Tôhoku Math. Journ.

$32(1980)$, 419-425.

\title{
REMARKS ON THE LIMIT SETS OF KLEINIAN GROUPS
}

\author{
KATSUMI INOUE
}

(Received July 12, 1979, revised November 19, 1979)

1. The so-called combination theorems of Maskit play an important role in the theory of Kleinian groups. In [6], Maskit proved that every function group can be constructed from elementary groups, quasi-Fuchsian groups and degenerate groups by using his combination theorems. Moreover, in [1] Abikoff and Maskit proved that every finitely generated Kleinian group can be constructed from elementary groups, degenerate groups and web groups in a similar manner. In this note we investigate the limit sets of Kleinian groups which are constructed by using the combination theorems.

2. Let $G$ be a Kleinian group and denote by $\Omega(G)$ and $\Lambda(G)$ the region of discontinuity and the limit set of $G$, respectively. We denote by $\mathrm{SL}^{\prime}$ the group of all the Möbius transformations. Consider a sequence $\left\{C_{n}\right\}$ of Jordan curves on $\hat{C}$ and a point $z \in \hat{C}$. We say that $\left\{C_{n}\right\}$ nests about $z$, if $C_{n+1}$ separates $z$ from $C_{n}$ for every natural number $n$ and if the sequence of spherical diameters of $\left\{C_{n}\right\}$ forms a null sequence.

Let $C$ be a Jordan curve on $\hat{C}$ and $\left\{g_{n}\right\}$ be a sequence of elements of SL'. We say that the sequence $\left\{g_{n}(C)\right\}$ converges to a point $z \in \hat{C}$, if there exists a point $x \in C$ so that $\left\{g_{n}(x)\right\}$ converges to $z$ and the sequence of spherical diameters of $\left\{g_{n}(C)\right\}$ forms a null sequence.

3. Let $G$ be a Kleinian group and let $H$ be a subgroup of $G$. A subset $S$ on $\hat{C}$ is called precisely invariant under $H$ in $G$, if $h(S)=S$ for every $h \in H$ and $g(S) \cap S=\varnothing$ for every $g \in G-H$. For a cyclic subgroup $H$ of $G$, a precisely invariant disc $B$ for $H$ is the interior of a closed topological disc $\bar{B}$ on $\hat{C}$, where $\bar{B}-\Lambda(H)$ is precisely invariant under $H$ in $G$ and $\bar{B}-\Lambda(H) \subset \Omega(G)$.

We use the combination theorems in the following forms.

Combination theorem I. Let $G_{1}$ and $G_{2}$ be two Kleinian groups and let $B_{i}(i=1,2)$ be a precisely invariant disc under $H$, a finite or a parabolic cyclic subgroup of both $G_{1}$ and $G_{2}$. Assume that $B_{1}$ and $B_{2}$ have the common boundary $\gamma$ and $B_{1} \cap B_{2}=\varnothing$. Let $G$ be the group generated by $G_{1}$ and $G_{2}$. Then we have the following: 
(I-1) $G$ is Kleinian.

(I-2) $G$ is the free product of $G_{1}$ and $G_{2}$ with the amalgamated subgroup $H$, where $G$ is often denoted by $G_{1} *_{H} G_{2}$.

(I-3) If $z \in \Lambda(G)-\bigcup_{g \in G} g\left(\Lambda\left(G_{1}\right) \cup \Lambda\left(G_{2}\right)\right)$, then there is a sequence $\left\{g_{n}\right\}$ of elements of $G$ so that $\left\{g_{n}(\gamma)\right\}$ nests about $z$.

(I-4) $g(\gamma)=\gamma$ if and only if $g \in H$.

(I-5) $g \in G_{i}-H$ and $g(\gamma) \cap \gamma \neq \varnothing$ if and only if $H$ is parabolic cyclic and $g \in N_{G_{i}}(H)$, where $N_{G_{i}}(H)$ denotes the normalizer of $H$ in $G_{i}$, for $i=1,2$.

(I-6) The cardinality of the set $g(\gamma) \cap \gamma$ is not greater than one for $g \in G-H$.

Combination theorem II. Let $G_{1}$ be a Kleinian group. For $i=1,2$, let $B_{i}$ be a precisely invariant disc for a finite or a parabolic cyclic subgroup $H_{i}$ of $G_{1}$ and let $\gamma_{i}$ be the boundary of $B_{i}$. Assume that $g\left(\bar{B}_{1}\right) \cap$ $\bar{B}_{2}=\varnothing$ for all $g \in G_{1}$. Let $f$ be a loxodromic element satisfying $f\left(\gamma_{1}\right)=\gamma_{2}$, $f\left(B_{1}\right) \cap B_{2}=\varnothing$ and $f^{-1} H_{2} f=H_{1}$. Let $G$ be the group generated by $G_{1}$ and $f$. Then we have the following:

(II-1) $G$ is Kleinian.

(II-2) Every relation in $G$ is a consequence of the relations in $G_{1}$ and the relation $f^{-1} H_{2} f=H_{1}$.

(II-3) If $z \in \Lambda(G)-\bigcup_{g \in G} g\left(\Lambda\left(G_{1}\right)\right)$, then there is a sequence $\left\{g_{n}\right\}$ of $G$ so that $\left\{g_{n}\left(\gamma_{1}\right)\right\}$ nests about $z$.

(II-4) $g\left(\gamma_{i}\right)=\gamma_{i}$ for $i=1,2$, if and only if $g \in H_{i}$.

(II-5) $g \in G_{1}-H_{i}$ and $g\left(\gamma_{i}\right) \cap \gamma_{i} \neq \varnothing$ for $i=1,2$, if and only if $H_{i}$ is parabolic cyclic and $g \in N_{G_{1}}\left(H_{i}\right)$.

(II-6) The cardinality of the set $g\left(\gamma_{i}\right) \cap \gamma_{i}$ is not greater than one for $i=1,2$, and for $g \in G-H_{i}$.

Proposition 1. Let $G=G_{1} *_{H} G_{2}$ and let $g \in G . \quad$ If $g(\gamma) \subset B_{j} \cup \Lambda(H)$ and $\left(g^{\prime} \circ g\right)(\gamma) \cap\left(\bar{B}_{j}-\Lambda(H)\right) \neq \varnothing$ for all $g^{\prime} \in G_{i}$ and for $i, j=1,2, i \neq j$, then $g \in G_{i}$.

Proof. We may assume $i=1$ and $j=2$. Since $g(\gamma) \cap B_{2} \neq \varnothing$, we have $g \in G-H$ by (I-4). So $g$ can be written in the normal form $g=$ $g_{n} \circ \cdots \circ g_{1}$, where either $g_{2 k} \in G_{1}-H$ and $g_{2 k+1} \in G_{2}-H$, or $g_{2 k} \in G_{2}-H$ and $g_{2 k+1} \in G_{1}-H$. This representation is not unique, but, as is well known, the number $n$ of factors on the right hand side of this representation is determined only by $g$. (See [3].) We call the number $n$ the length of $g$ and denote it by $L(g)$.

By our assumption, we see $g_{n} \in G_{1}-H$. Assume $L(g) \geqq 2$. Since $\left(g^{\prime} \circ g\right)(\gamma) \subset \bar{B}_{2}-\Lambda(H)$ for all $g^{\prime} \in G_{1}$ and since $g_{n}^{-1} \in G_{1}$, we have $\left(g_{n}^{-1} \circ g\right)(\gamma)=$ 
$\left(g_{n-1} \circ \cdots \circ g_{1}\right)(\gamma) \subset \bar{B}_{2}-\Lambda(H)$. This means $g_{n-1} \in G_{1}-H$, which contradicts the choice of $g_{1}, \cdots, g_{n}$. Hence we have $L(g)=1$. Therefore $g=g_{1} \in G_{1}$ and we are done.

Let $G$ be a Kleinian group constructed from $G_{1}$ and $f$ by using Combination theorem II. Every element $g \in G$ can be represented, not necessarily uniquely, as a word in the form $g=f^{a_{n+1}} \circ g_{n} \circ \cdots \circ g_{1} \circ f^{a_{1}}$, where $g_{1}, \cdots, g_{n} \in G_{1}$ and $a_{1}, \cdots, a_{n+1}$ are integers. In this representation we assume that $a_{i+1} \geqq 0$ whenever $a_{i}>0$ and $g_{i} \in H_{2}$, and that $a_{i+1} \leqq 0$ whenever $a_{i}<0$ and $g_{i} \in H_{1}$.

The following is due to Maskit [5].

Proposition 2. Let $G$ be a Kleinian group constructed from $G_{1}$ and $f$ by using Combination theorem II. For $g \in G$, assume $g=f^{a_{n+1}}$ 。 $g_{n} \circ \ldots \circ g_{1} \circ f^{a_{1}}$. Then $a_{n+1}>0$ (resp. $a_{n+1}<0$ ) implies $g\left(\gamma_{1}\right) \subset \bar{B}_{2}$ (resp. $\left.g\left(\gamma_{1}\right) \subset B_{1}\right)$.

4. Let $G$ be a Kleinian group which is constructed from $G_{1}, \cdots, G_{s}$ and $f_{1}, \cdots, f_{t}$ by using Combination theorems I and II, where $s+t \geqq 2$. Put $\Lambda_{N}(G)=\Lambda(G)-\mathbf{U}_{g \in G} g\left(\mathbf{U}_{i=1}^{s} \Lambda\left(G_{i}\right)\right)$. Then we have the following.

THEOREM 1. Under the above notations, let $G=G_{1} *_{H} G_{2}$ and $\lambda \in \Lambda(G)$ and let $\gamma$ be the common boundary of precisely invariant discs under $H$. Assume that there is a sequence $\left\{g_{n}\right\}$ of elements of $G$ such that $\left\{g_{n}(\gamma)\right\}$ nests about $\lambda$. Then $\lambda \in \Lambda_{N}(G)$.

Proof. Otherwise, then there are $g_{0} \in G$ and $\lambda_{0} \in \Lambda\left(G_{i}\right)$ such that $\left\{\left(g_{0} \circ g_{n}\right)(\gamma)\right\}$ nests about $g_{0}(\lambda)=\lambda_{0} \in \Lambda\left(G_{i}\right)$ for $i=1$ or 2 . We may assume $i=1$ and $\infty \in \Omega(G)$. We first observe $\lambda_{0} \notin \mathbf{U}_{g \in G_{1}} g(\Lambda(H))$. If not, then there are $\widetilde{g}_{0} \in G_{1}$ and $\tilde{\lambda}_{0} \in \Lambda(H)$ so that $\widetilde{g}_{0}\left(\lambda_{0}\right)=\widetilde{\lambda}_{0} \in \Lambda(H) \subset \gamma$. It shows that $\left\{\left(\widetilde{g}_{0} \circ g_{0} \circ g_{n}\right)(\gamma)\right\}$ nests about $\tilde{\lambda}_{0} \in \gamma$ and the cardinality of the set $\left(\widetilde{g}_{0} \circ g_{0} \circ g_{n}\right)(\gamma) \cap \gamma$ is greater than one for a sufficiently large $n$. It clearly contradicts (I-6). Thus we have $\lambda_{0} \in \Lambda\left(G_{1}\right)-\mathbf{U}_{g \in G_{1}} g(\Lambda(H))$.

Since $\lambda_{0} \in \Lambda\left(G_{1}\right)$, there is a sequence $\left\{h_{n}\right\}$ of elements of $G_{1}$ such that the sequence of Jordan curves $\left\{h_{n}(\gamma)\right\}$ converges to $\lambda_{0}$. By the definition of precisely invariant discs, we see that $\lambda_{0}$ is contained in the exterior of $h_{n}\left(B_{1}\right)$ for all $n$. We set $\widetilde{g}_{n}=g_{0} \circ g_{n}$. By choosing subsequences of $\left\{h_{n}\right\}$ and $\left\{\widetilde{g}_{n}\right\}$, again denoted by $\left\{h_{n}\right\}$ and $\left\{\widetilde{g}_{n}\right\}$, respectively, we have that $h_{n}\left(B_{1}\right)$ is contained in the intersection of $\operatorname{Int} \widetilde{g}_{n-1}(\gamma)$ and $\operatorname{Ext} \widetilde{g}_{n}(\gamma)$, where Int $\widetilde{g}_{n-1}(\gamma)$ (resp. Ext $\widetilde{g}_{n}(\gamma)$ ) means the interior of $\widetilde{g}_{n-1}(\gamma)$ (resp. the exterior of $\left.\widetilde{g}_{n}(\gamma)\right)$.

Now, let $m$ be an arbitrary fixed integer. Since $g \in H h_{m}^{-1} \subset G_{1}$ implies $\left(g \circ h_{m}\right)\left(B_{1}\right)=B_{1}$ and $g \in G_{1}-\left\{H h_{m}^{-1}\right\}$ implies $\left(g \circ h_{m}\right)\left(B_{1}\right) \subset B_{2}$ and since $g\left(\lambda_{0}\right) \in$ 
$\Lambda\left(G_{1}\right)$ for all $g \in G_{1}$, we have $\left(g \circ \widetilde{g}_{m}\right)(\gamma) \subset \vec{B}_{2}-\Lambda(H)$ for all $g \in G_{1}$. Thus Proposition 1 shows $\widetilde{g}_{m} \in G_{1}$ and we see $\left\{\widetilde{g}_{n}\right\} \subset G_{1}$. If $\widetilde{g}_{n} \in G_{1}$, then $\lambda_{0}$ is contained in the exterior of $\widetilde{g}_{n}\left(B_{1}\right)$, which contradicts the fact that $\left\{\widetilde{g}_{n}(\gamma)\right\}$ nests about $\lambda_{0}$. This completes the proof of Theorem 1 .

THEOREM 2. Let $G$ be a Kleinian group constructed from $G_{1}$ and $f$ by using Combination theorem II and let $\lambda \in \Lambda(G)$. Assume that there is a sequence $\left\{g_{n}\right\}$ of elements of $G$ such that $\left\{g_{n}\left(\gamma_{1}\right)\right\}$ nests about $\lambda$. Then $\lambda \in \Lambda_{N}(G)$.

Proof. Assume the contrary. Then there are $g_{0} \in G$ and $\lambda_{0} \in \Lambda\left(G_{1}\right)$ such that $\left\{\left(g_{0} \circ g_{n}\right)\left(\gamma_{1}\right)\right\}$ nests about $g_{0}(\lambda)=\lambda_{0} \in \Lambda\left(G_{1}\right)$. We may assume $\infty \in \Omega\left(G_{1}\right)$. Since $\lambda_{0} \in \Lambda\left(G_{1}\right)$, there is a sequence $\left\{h_{n}\right\}$ of elements of $G_{1}$ such that $\left\{h_{n}\left(\gamma_{1}\right)\right\}$ converges to $\lambda_{0}$ and $\lambda_{0}$ is contained in the exterior of $h_{n}\left(B_{1}\right)$ for all $n$.

Set $B_{3}=\hat{C}-\left(\bar{B}_{1} \cup \bar{B}_{2}\right)$ and $\widetilde{g}_{n}=g_{0} \circ g_{n}$. Then we have $\lambda_{0} \in B_{3}$, $\widetilde{g}_{n}\left(\gamma_{1}\right) \subset B_{3}$ and $h_{n}\left(B_{1}\right) \subset B_{3}$ for sufficiently large $n$ 's. Choosing subsequences of $\left\{h_{n}\right\}$ and $\left\{\widetilde{g}_{n}\right\}$, again denoted by $\left\{h_{n}\right\}$ and $\left\{\widetilde{g}_{n}\right\}$, respectively, we have that $h_{n}\left(B_{1}\right)$ is contained in the intersection of Int $\widetilde{g}_{n-1}\left(\gamma_{1}\right)$ and Ext $\widetilde{g}_{n}\left(\gamma_{1}\right)$.

Now, let $m$ be an arbitrary fixed integer and let $\widetilde{g}_{m}=f^{a_{k+1}} \circ g_{m, k} \circ \ldots \circ$ $g_{m, 1} \circ f^{a_{1}}$, where $g_{m, 1}, \cdots, g_{m, k} \in G_{1}$ and $a_{1}, \cdots, a_{k+1}$ are integers. Since $\widetilde{g}_{m}\left(\gamma_{1}\right) \subset B_{3}$, we have $a_{k+1}=0$ by Proposition 2 and $\widetilde{g}_{m}=g_{m, k} \circ \cdots \circ g_{m, 1} \circ f^{a_{1}}$.

Next we show $a_{k}=0$. If $g \in H h_{m}^{-1} \subset G_{1}$, then $\left(g \circ h_{m}\right)\left(B_{1}\right)=B_{1}$. If $g \in$ $G_{1}-\left\{H h_{m}^{-1}\right\}$, then $\left(g \circ h_{m}\right)\left(B_{1}\right) \subset B_{3}$. Since $g\left(\lambda_{0}\right) \in B_{3}$ for all $g \in G_{1}$, we see $\left(g \circ \widetilde{g}_{m}\right)\left(\gamma_{1}\right) \subset B_{3}$ for all $g \in G_{1}$. By Proposition 2, we have $a_{k}=0$, so $\widetilde{g}_{m}=$ $g_{m, 1} \circ f^{a_{1}}$. By repeating this procedure, we have $a_{1}=0$ and then $\widetilde{g}_{m}=$ $g_{m, 1} \in G_{1}$. This contradicts the fact that $\left\{\widetilde{g}_{n}\left(\gamma_{1}\right)\right\}$ nests about $\lambda_{0}$. This completes the proof of Theorem 2.

REMARK. Let $G=G_{1} *_{H} G_{2}$ and $\lambda \in \Lambda_{N}(G)$. Then there exists a sequence $\left\{g_{n}\right\}$ of elements of $G$ so that $\left\{g_{n}(\gamma)\right\}$ nests about $\lambda$. Theorem 1 and (I-3) show that the set $\Lambda_{N}(G)$ coincides with the set of all limit points which are nested by the translates of $\gamma$ under $G$. We have a similar result in the case of Theorem 2.

Now we prove the following.

Theorem 3. Let $G$ be a Kleinian group constructed from $G_{1}, \cdots, G_{s}$ and $f_{1}, \cdots, f_{t}$ by using Combination theorems I and II, where $s+t \geqq 2$. Then the cardinality of $\Lambda_{N}(G)$ is either zero, two or that of a continuum. lemmas.

In order to prove Theorem 3, it suffices to show the following two 
Lemma 1. Let $G=G_{1} *_{H} G_{2}$. Then either $\Lambda_{N}(G)$ is empty or has the power of a continuum.

Proof. Assume that $\Lambda_{N}(G)$ is a non-empty finite set. Since $\Lambda_{N}(G)$ is invariant under the action of $G$, we see that $\Lambda_{N}(G)$ is an infinite set whenever $G$ is not elementary. Thus $G$ and $G_{i}$ are elementary groups for $i=1,2$. By the classification of elementary groups, $G$ and $G_{i}$ $(i=1,2)$ must be those whose signatures are $(0,4 ; 2,2,2,2)$ and $(0,3 ; 2,2, \infty)$, respectively. But in this case, $\Lambda_{N}(G)$ is an empty set. Therefore, $\Lambda_{N}(G)$ is an empty set or $\Lambda_{N}(G)$ is an infinite set.

Assume that $\Lambda_{N}(G)$ is an infinite set. Let $\infty \in \Omega(G)$. For $\lambda_{0} \in \Lambda_{N}(G)$, there is an element $g^{*} \in G$ such that $\lambda_{0} \in \operatorname{Int} g^{*}(\gamma)$. Set $U^{*}=\operatorname{Int} g^{*}(\gamma)$ and take two distinct points $\lambda_{1}$ and $\lambda_{2}$ in $U^{*} \cap \Lambda_{N}(G)$. Then we can choose $g_{0}, g_{1}$ and $g_{2} \in G$ in such a way that $\bar{U}_{i} \subset U^{*}$ and $\bar{U}_{i} \cap \bar{U}_{j}=\varnothing$ for $i, j=0,1$ and $2(i \neq j)$ and that $\lambda_{i} \in U_{i}$, where $U_{i}=\operatorname{Int} g_{i}(\gamma)$. Take this procedure for each $\lambda_{i}$. $\quad(i=0,1$ and 2.)

Let $\Gamma$ be the subset of all elements of $G$, which are chosen under these procedures. Choose a point $x_{0} \in U^{*} \cap\left(\bigcap_{g \in \Gamma-\left\{g^{*}\right\}} \operatorname{Ext} g(\gamma)\right)$. For each $g \in \Gamma-\left\{g^{*}\right\}$, we set $D(g)=\operatorname{Int} g(\gamma)$. Let $w(g)$ be a path from $x_{0}$ to a boundary point of $D(g)$, where $w(g)$ meets each translate of $\gamma$ under $\Gamma$ at one point at most. Denote by $\nu(g)$ the cardinality of the set $w(g) \cap$ $\left(\bigcup_{g^{\prime} \in \Gamma} g^{\prime}(\gamma)\right)$ and set $D_{n}=\bigcup_{n=\nu\left(g^{\prime}\right)} \operatorname{Int} g^{\prime}(\gamma)$ for $n=1,2, \cdots$. Then we have $\bar{D}_{n+1} \subset D_{n}$ for all $n$. Let $D=\bigcap_{n=1}^{\infty} \bar{D}_{n}$, which is clearly closed.

Next we show $D \subset \Lambda_{N}(G)$. For all $\lambda_{0} \in D$, we have $\lambda_{0} \in \bar{D}_{n}$ for all $n$ and, moreover, $\lambda_{0} \in D_{n}$ for all $n$. Then there exists a sequence $\left\{g_{n}\right\}$ of elements of $\Gamma$ such that $\left\{g_{n}(\gamma)\right\}$ nests about $\lambda_{0}$. By Theorem 1 we have $D \subset \Lambda_{N}(G)$. Since $D$ is dense, $D$ is a perfect set. Hence the power of $D$ is that of a continuum. This completes the proof of Lemma 1.

LEMMA 2. Let $G$ be a Kleinian group constructed from $G_{1}$ and $f$ by using Combination theorem II. Then $\Lambda_{N}(G)$ consists of just two points or has the power of a continuum.

Proof. Since $f$ is loxodromic, we may suppose that $\lambda_{1}$ and $\lambda_{2}$ are the attractive and the repelling fixed points of $f$, respectively. Since $\left\{f^{n}\left(\gamma_{1}\right)\right\}$ nests about $\lambda_{1}$ and $\left\{f^{-n}\left(\gamma_{1}\right)\right\}$ nests about $\lambda_{2}$, two points $\lambda_{1}$ and $\lambda_{2}$ are contained in $\Lambda_{N}(G)$ by Theorem 2 . Therefore we see that $\Lambda_{N}(G)$ consists of at least two points. If $G$ is not elementary, $\Lambda_{N}(G)$ contains infinitely many points. Thus $\Lambda_{N}(G)$ consists of two points only if $G$ is elementary. By Ford [2], if $\Lambda_{N}(G)$ consists of two points, then $G$ is a cyclic group generated by a loxodromic element, or a group generated by a loxodromic element and an elliptic element, which have the common 
fixed points. By an argument similar to that of the proof of Lemma 1, we see that $\Lambda_{N}(G)$ has the power of a continuum whenever $G$ is not elementary. This completes the proof of Lemma 2.

It is obvious that Theorem 3 can be established by Lemmas 1 and 2 .

THEOREM 4. Let $G$ be a Kleinian group constructed from $G_{1}, \cdots, G_{s}$ and $f_{1}, \cdots, f_{t}$ by using Combination theorems I and II, where $s+t \geqq 2$. If $\Lambda_{N}(G)=\varnothing$, then (i) $s=2$ and $t=0$ (i.e., $G$ is constructed from $G_{1}$ and $G_{2}$ by using Combination theorem I). (ii) $G$ has the signature $(0,4 ; 2,2,2,2)$ and (iii) $G_{i}(i=1,2)$ has the signature $(0,3 ; 2,2, \infty)$. Conversely, the properties (i), (ii) and (iii) imply $\Lambda_{N}(G)=\varnothing$.

Proof. The second assertion of Theorem 4 can be seen in the proof of Lemma 1. So we prove the first assertion.

First we suppose that Combination theorem II is used in the last step of the construction of $G$ from $G_{1}, \cdots, G_{s}$ and $f_{1}, \cdots, f_{t}$. Then $G$ is constructed from $G^{\prime}$ and $f_{t}$ by using Combination theorem II, where $G^{\prime}$ is the group constructed from $G_{1}, \cdots, G_{s}$ and $f_{1}, \cdots, f_{t-1}$. We set $\widetilde{\Lambda}_{N}(G)=\Lambda(G)-\bigcup_{g \in G} g\left(\Lambda\left(G^{\prime}\right)\right)$. By definition, we see $\Lambda_{N}(G) \supset \widetilde{\Lambda}_{N}(G)$ and $\Lambda\left(\left\langle f_{t}\right\rangle\right) \subset \tilde{\Lambda}_{N}(G) \subset \Lambda_{N}(G)$, where $\left\langle f_{t}\right\rangle$ is the cyclic group generated by $f_{t}$. Since $\Lambda\left(\left\langle f_{t}\right\rangle\right)$ consists of just two points, we see that $\Lambda_{N}(G)$ contains at least two points. This contradicts the assumption $\Lambda_{N}(G)=\varnothing$. Hence we conclude that $G$ is constructed by using Combination theorem $\mathrm{I}$ in the last step.

We set $G_{2}^{\prime}=G_{s}$ and $G_{1}^{\prime}$ the group constructed from $G_{1}, \cdots, G_{s-1}$ and $f_{1}, \cdots, f_{t}$. Then $G$ is the group constructed from $G_{1}^{\prime}$ and $G_{2}^{\prime}$ by using Combination theorem I. Hence we have $\tilde{\Lambda}_{N}(G)=\Lambda(G)-\bigcup_{g \in G} g\left(\Lambda\left(G_{1}^{\prime}\right) \cup\right.$ $\left.\Lambda\left(G_{2}^{\prime}\right)\right) \subset \Lambda_{N}(G)$.

Assume $N_{G_{i}^{\prime}}(H) \varsubsetneqq G_{i}^{\prime}$ for $i=1$ or 2 . We may assume $i=1$. Then there exists an element $g_{1} \in G_{1}^{\prime}-N_{G_{i}^{\prime}}(H)$. For an arbitrary fixed element $g_{2} \in G_{2}^{\prime}-H$, we set $g=g_{2} \circ g_{1}$. Since $g_{1}$ is not contained in $N_{G_{1}^{\prime}}(H)$, we see $\left(g_{2} \circ g_{1}\right)\left(\bar{B}_{1}\right) \subset B_{1}$ by (I-5). This means that $g$ is a loxodromic element, whose fixed points are contained in $\tilde{\Lambda}_{N}(G)$. This contradicts the fact $\Lambda_{N}(G)=\varnothing$. Thus this case does not occur.

Therefore we have $N_{G_{i}^{\prime}}(H)=G_{i}^{\prime}$ for $i=1$ and 2 . In this case $G$ has the signature $(0,4 ; 2,2,2,2)$ and $G_{i} \quad(i=1,2)$ has the signature $(0,3 ; 2,2, \infty)$. It is well known that the elementary group with the signature $(0,3 ; 2,2, \infty)$ cannot be decomposed into simpler groups by using the combination theorems. This completes the proof of Theorem 4 .

REMARK. By Theorem 4, we see that $\Lambda_{N}(G) \varsubsetneqq \Lambda(G)$ if $\Lambda_{N}(G)=\varnothing$. The proof of Lemma 2 shows that $\Lambda_{N}(G)=\Lambda(G)$ if $\Lambda_{N}(G)$ consists of just 
two points. In the case where $\Lambda_{N}(G)$ has the power of a continuum, there are also groups $G$ whose limit sets coincide with $\Lambda_{N}(G)$. These groups are those which are constructed from $G_{1}, \cdots, G_{s}$ and $f_{1}, \cdots, f_{t}$ by using Combination theorems I and II, where $G_{i}(i=1, \cdots, s)$ is a finite cyclic group. The most conspicuous example of these groups is a Schottky group.

\section{REFERENCES}

[1] W. Abikoff and B. Maskit, Geometric decompositions of Kleinian groups, Amer. J. Math. 99 (1977), 687-697.

[2] L. R. Ford, Automorphic Functions, 2nd ed. Chelsea, New York, 1951.

[3] R. C. Lyndon AND P. E. Schupp, Combinatorial Group Theory, Springer-Verlag, BerlinHeidelberg-New York, 1977.

[4] B. MASKIT, On Klein's combination theorem, Trans. Amer. Math. Soc. 120 (1965), 499-509.

[5] B. MASKit, On Klein's combination theorem II, Trans. Amer. Math. Soc. 131 (1968), 32-39.

[6] B. MASKIT, Decomposition of certain Kleinian groups, Acta Math. 130 (1973), 243-263.

Mathematical Institute

TÔHOKU UNIVERSITY

SENDAI, 980 JAPAN 
